\title{
A MAGNETIC FIELD STRENGTH $V S$. TEMPERATURE RELATION IN SUNSPOTS
}

\author{
GREG KOPP and DOUGLAS RABIN \\ National Solar Observatory, National Optical Astronomy Observatories, * \\ P.O. Box 26732, Tucson, AZ 85726, U.S.A.
}

\begin{abstract}
The near infrared presents several new and powerful advantages in the diagnostics of sunspot atmospheres: (1) increased magnetic sensitivity in Zeeman-split lines, (2) increased sensitivity of umbral brightness to temperature, and (3) reduced scattered light and seeing disturbances due to atmospheric turbulence. This has revealed a strong and consistent relationship between sunspot brightness and magnetic field strength.

We have made spatial/spectral observations of sunspots in the highly sensitive $(g=3) \mathrm{Fe} \mathrm{I} \mathrm{line}$ at $\lambda=1.5649 \mu \mathrm{m}$ to compare field strengths with continuum intensities. We find a characteristic but nonlinear relationship between magnetic field strength, $B$, and brightness temperature, $T_{b}$, in sunspots. In umbrae there is an approximately linear relation between $B^{2}$ and $T_{b}$.
\end{abstract}

Key words: infrared: stars - Sun: magnetic fields - sunspots

\section{Introduction}

There are three primary reasons to observe in the infrared rather than the visible when making measurements of sunspot magnetic fields. The first is the extra magnetic sensitivity afforded by infrared lines, since Zeeman splitting as a fraction of linewidth increases linearly with wavelength. The second is the reduced stray light in the infrared, where instrumental scatter is lower (Pierce, 1991). Finally, the effects of stray light on intensity measurements are smaller in the infrared because of the greater umbral brightness. Whereas umbrae are typically $10 \%$ as bright as the mean quiet Sun in the visible, they are roughly $50 \%$ as bright in the near infrared, greatly reducing the effects of contamination by stray light from the surrounding photosphere.

We present sunspot observations using the magnetically-sensitive (Landé $g=3$ ) Fe I line at $1.5649 \mu \mathrm{m}$. First used for magnetic work in 1975 (Harvey and Hall), this line has been useful in determining the magnetic fields of fluxtubes (Stenflo, Solanki and Harvey, 1987), plages (Rabin, 1992) and sunspots (McPherson, Lin, and Kuhn, 1992; Kopp and Rabin, 1992).

Zeeman splitting of this line is roughly three times that of high- $g$ visible lines. Figure 1 shows an example of the splitting in this line for a 2,600 G sunspot. The magnetic field strength, not just magnetic flux, can be determined from the completely split $\pi$ - and $\sigma$-components of the line; thus, these field strength determinations are insensitive to field inclination. The neighboring $g_{\mathrm{eff}}=1.53$ line at $1.5653 \mu \mathrm{m}$ (Solanki, Biémont and Mürset, 1990) is obscured by umbral OH lines (Wallace and Livingston, 1992), making it useful for spectral calibration but not as an alternate measurement of field strength.

\footnotetext{
* Operated by the Association of Universities for Research in Astronomy, Inc., under cooperative agreement with the National Science Foundation.
} 


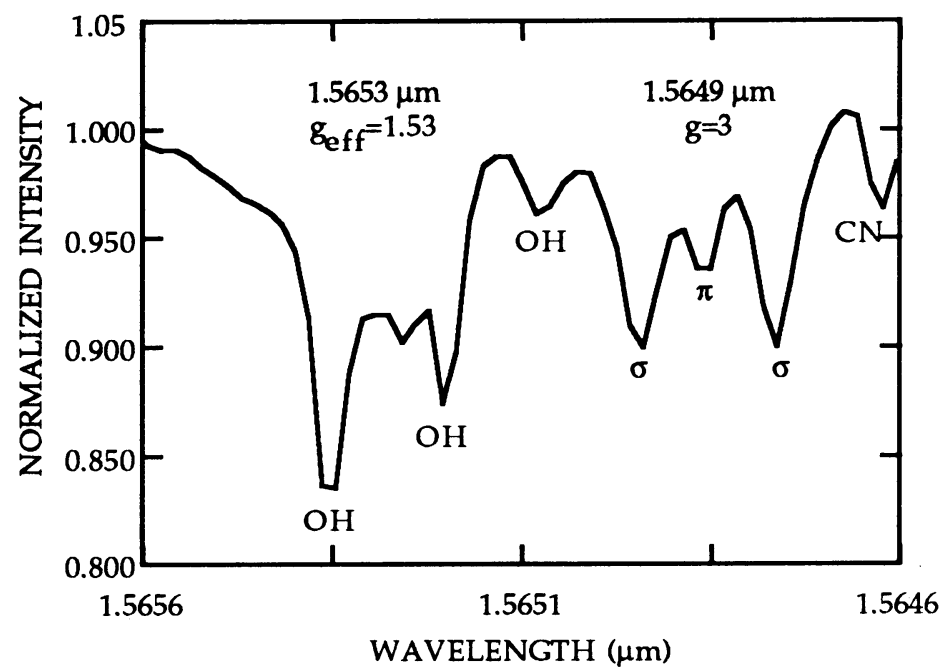

Fig. 1. Umbral spectrum of the Fe I lines at $1.5649 \mu \mathrm{m}(g=3)$ and at $1.5653 \mu \mathrm{m}$ $\left(g_{\text {eff }}=1.53\right)$ for a field strength of $2,600 \mathrm{G}$. Umbral OH lines obscure the $g_{\text {eff }}=1.53$ line.

\section{Observations}

Several sunspots, each close to disk center $(\mu=\cos \theta \geq 0.75)$, were observed during two observing runs in May 1991 and January 1992. The observations were made using the McMath East Auxiliary Telescope and the 13.8-m vertical spectrograph. The KPNO $58 \times 62 \mathrm{InSb}$ infrared array (Fowler et al., 1987) was used to take spatial/spectral images of the $1.5649 \mu \mathrm{m}$ line by scanning sunspots across the spectrograph. Large spots required multiple-swath scans. Swath registration was done using large-field K-line images taken before and after each scan. The spatial scale was $0.85^{\prime \prime}$ per pixel, although the resolution was seeing-limited to roughly $2^{\prime \prime}$. The spectral resolving power was limited by pixel sampling to $\sim 35,000$.

Each spatial/spectral image, created by co-adding twenty 50 -ms frames, corresponds to one chord in a sunspot admitted into the slit. The magnetic field strength is determined as a function of position across the spot from fits of blended Gaussians to the line profile. The error on the position of each completely split component of the line is typically $\pm 20 \mathrm{G}$; the splitting is incomplete for fields weaker than $1,000 \mathrm{G}$. The continuum intensity is determined from a line-free region of the spectrum. A two-dimensional image of the continuum brightness of the spot and of its magnetic-field can be reconstructed from the spatial/spectral images in all of the swaths.

\section{Results and Discussion}

We find a non-linear relation between magnetic field strength, $B$, and continuum intensity, $I_{c}$, for the observed sunspots, although the relation is nearly linear within 


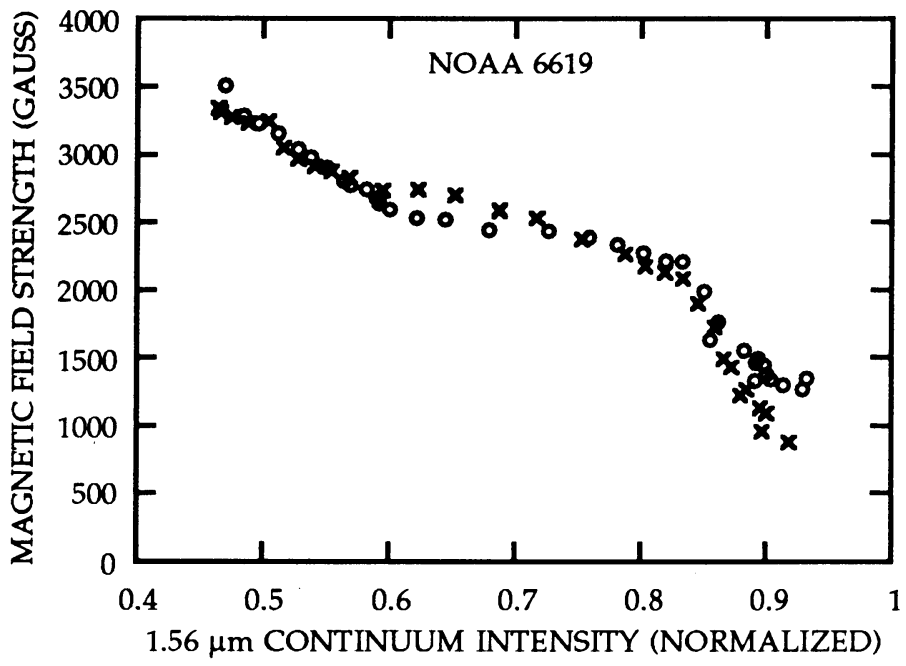

Fig. 2. Magnetic field strength vs. continuum intensity across a diameter of NOAA 6619, 13 May 1991. Opposite sides of spot center are identified by different symbols.

umbrae. Figure 2 shows this characteristic relation for a single large spot. The relationship appears linear in three distinct regions: for $B \gtrsim 2,500 \mathrm{G}$ (umbrae), field strength drops sharply with increasing intensity; for $2,000<B<2,500 \mathrm{G}$ (near the umbra/penumbra boundary), the field strength decreases little with increasing intensity; below 2,000 G the field strength again drops quickly with larger intensities. This region near the penumbral boundary has more scatter and the magnetic resolution of these observations begins to fail for $B \lesssim 1,000 \mathrm{G}$.

Similar behavior is seen in other large spots. Small spots that do not reach field strengths larger than $2,500 \mathrm{G}$ do not show the linear $B$ vs. $I_{c}$ dependence in this region (the intensity profile of a small spot may also be affected by blurring). A superposition of six spots observed in May 1991 is shown in Figure 3. Although no attempt was made to register the profiles of different spots, the same threecomponent behavior is evident in the superposition, although the scatter is larger than for a single spot. More recent data confirm this behavior.

Gurman and House (1981) proposed a linear $B$ vs. $I_{c}$ relation on the basis of visible-light observations, but there is apparently no magnetohydrodynamic basis for expecting linearity. Del Toro Iniesta et al., 1991, suggest instead that a linear relationship holds between $B^{2}$ and the temperature $T_{b}$. If we plot $B^{2} v s . T_{b}$, which is nearly proportional to $I_{c}$ for the intensities observed near $1.6 \mu \mathrm{m}$, we again find a three-component shape, not a single linear relation.

A single linear relation between $B$ or $B^{2}$ and $I_{c}$ or $T_{b}$ is inconsistent with most models of sunspot field profiles. The magnetic field in sunspots is typically modeled as a smooth function of distance from spot center, parameterized by only one length scale, usually the penumbral radius $r_{p}$ (Beckers and Schröter 1969; Gurman and House 1981; Adam 1990). In contrast, the observed continuum intensity has 


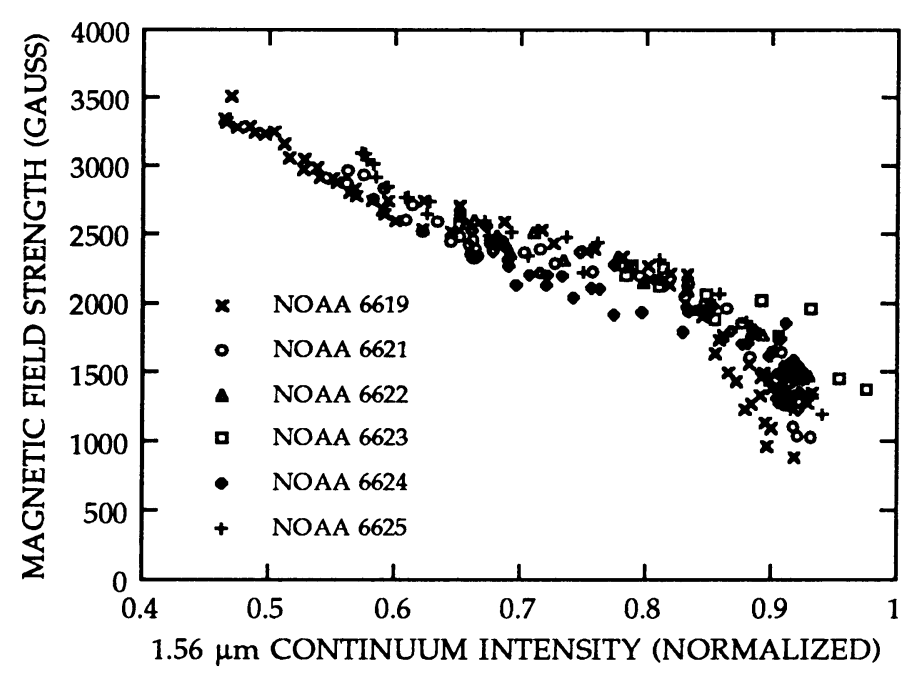

Fig. 3. Magnetic field strength vs. continuum intensity across 6 sunspots observed on 13 May 1991.

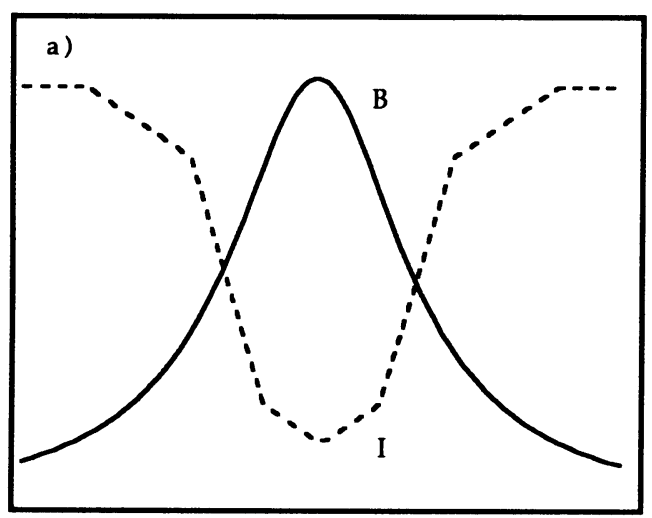

SPATIAL EXTENT

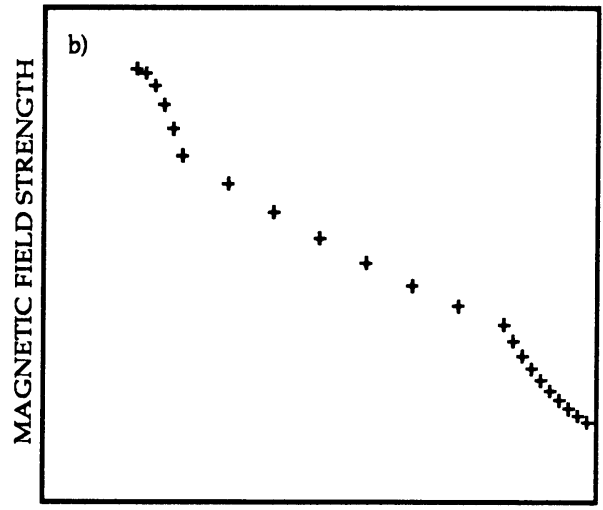

CONTINUUM INTENSITY

Fig. 4. a) Profiles of a hypothetical smooth magnetic field (solid curve) and a quickly changing intensity (dashed). b) Corresponding three-component nonlinear relation between field strength and intensity, similar to that observed. 


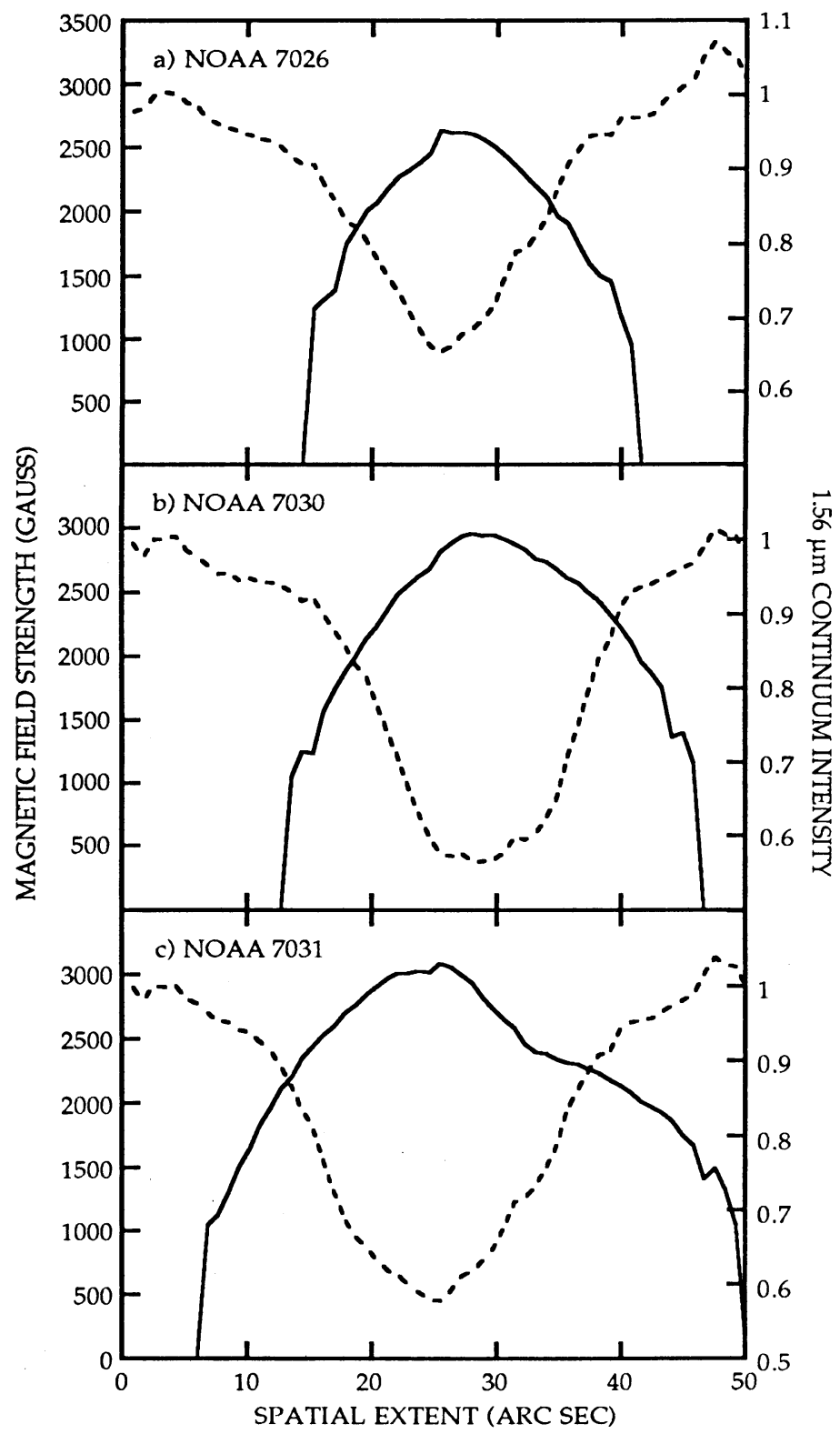

Fig. 5. Magnetic field (solid) and intensity (dashed) profiles for 3 spots observed on 26 Jan. 1992. Figures $5 a$ and $5 b$ show the field strength changing smoothly across the umbra/penumbra boundary, seen as a sudden change in slope of the intensity. Figure $5 c$ shows that this is not always the case, as the boundary at $32^{\prime \prime}$ on the abscissa is more evident in the magnetic field profile than in the intensity. Magnetic fields are only determined above $1,000 \mathrm{G}$. 
rapid spatial variations, creating the effect of a sharp umbra/penumbra boundary and outer penumbral boundary. Smooth changes in $B$, in conjunction with sudden changes in $I_{c}$, naturally result in plots similar to those observed, because the magnetic field changes only slowly while the intensity changes rapidly near the umbra/penumbra boundary (see Fig. 4).

However, the observed field does not always vary smoothly with distance from spot center. Occasionally the magnetic field shows a sharper change at the umbra/penumbra transition than the intensity does, implying the magnetic field is affected by the transition. Figures $5 a$ and $5 b$ show typical cases where the magnetic field varies smoothly across the umbra/penumbra boundary, which is seen as a change in slope of the continuum intensity. Figure $5 c$, however, shows a spot where the magnetic field strength undergoes a marked change in slope near the transition, at $32^{\prime \prime}$ on the abscissa, while the intensity shows only a small change. This result suggests that models of sunspot magnetic fields can be better parametrized by length scales including at least the umbral and penumbral radii $r_{u}$ and $r_{p}$.

We conclude that the relation between magnetic field and continuum intensity or temperature is not linear, but behaves according to the three-component plots shown in Figures 2 and 3. The shape of this curve is largely a consequence of large variations in intensity with radial distance from spot center accompanied by small changes in magnetic field. Sunspot magnetic fields, however, are not always smooth across umbra/penumbra boundaries, and models of the fields further depend upon length scales $r_{u}$ and $r_{p}$. Future work, combining better spectral resolution with full Stokes profiles of the $1.5649 \mu \mathrm{m}$ line, will allow the determination of field orientation and filling factor as well as a sensitive measurement of field strength, allowing for vector magnetometry beyond the edge of sunspot penumbrae.

\section{Acknowledgements}

We thank D. Jaksha, J. Wagner, and C. Plymate for their dedication during the observations. This work was partially supported by NASA under Task 170-38-5101-10.

\section{References}

Adam, M. G.: 1990, Solar Phys. 125, 37.

Beckers, J. M., and Schröter, E. H.: 1969, Solar Phys. 10, 384.

del Toro Iniesta, J.C., Martinez Pillet, V., and Vasquez, M.: 1991, in L. November (ed.), Solar Polarimetry ( Proc. 11th Sacramento Peak Workshop), Sunspot, New Mexico, p. 224.

Fowler, A. M., Probst, R. G., Britt. J. P., Joyce, R. R., and Gillett, F. C.: 1987, Opt. Eng. 26, 232.

Gurman, J., and House, L.: 1981, Solar Phys. 71, 5.

Harvey, J. W., and Hall, D.: 1975, Bull. Amer. Astron. Soc. 7, 459.

Kopp, G., and Rabin, D.: 1992, Solar Phys. 141, 253.

McPherson, M. R., Lin, H., and Kuhn, J. R.: 1992, Solar Phys. 139, 255.

Pierce, K.: 1991, Solar Phys. 133, 215.

Rabin, D.: 1992, Astrophys. J. $391,832$.

Solanki, S. K., Biémont, E., and Mürset, U.: 1990, Astron. A strophys. Suppl. 83, 307.

Stenflo, J. O., Solanki, S. K., and Harvey, J. W.: 1987, A stron. A strophys. 173, 167.

Wallace, L., and Livingston, W.: 1992, An Atlas of a Dark Sunspot Umbral Spectrum from 1970 to $8640 \mathrm{~cm}^{-1}$ (1.16 to $5.1 \mu \mathrm{m}$ ), Kitt Peak National Observatory, Tucson. 